\title{
CORRECTION
}

\section{Correction to: Aryl hydrocarbon receptor signaling promotes ORMDL3-dependent generation of sphingosine-1-phosphate by inhibiting sphingosine-1-phosphate lyase}

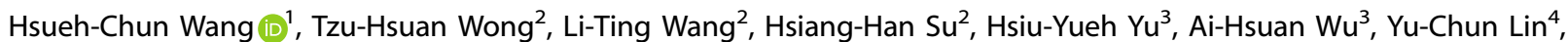
Hua-Ling Chen ${ }^{4}$, Jau-Ling Suen ${ }^{2,5,6}$, Shih-Hsien Hsu ${ }^{2,5}$, Li-Chen Chen ${ }^{3,7,8}$, Yufeng Zhou ${ }^{9,10}$ and Shau-Ku Huang ${ }^{2,4,5,9,11}$

Cellular \& Molecular Immunology (2018) 15:870; https://doi.org/10.1038/s41423-018-0040-0

Correction to: Cellular \& Molecular Immunology https://doi.org/ 10.1038/s41423-018-0022-2; published online: 23 March 2018
In this article, published online 23 March 2018, the affiliation 10 of Zhou Y was incorrect. The affiliation should be "Children's Hospital and Institute of Biomedical Sciences, Fudan University. Key Laboratory of Neonatal Disease, Ministry of Health, 201102 Shanghai, China." The authors regret the errors.

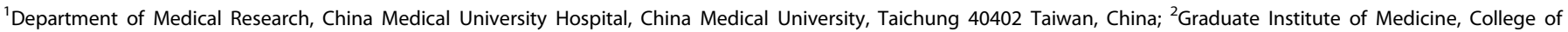

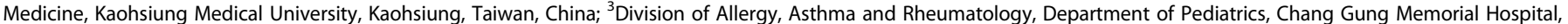

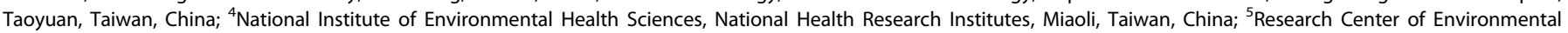

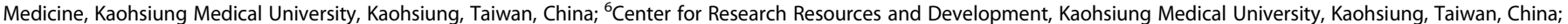

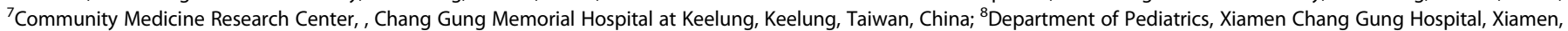

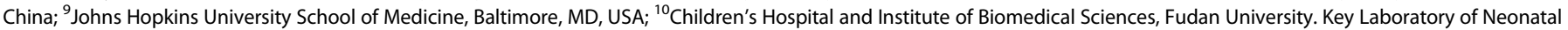
Disease, Ministry of Health, 201102 Shanghai, China and ${ }^{11}$ Lou-Hu Hospital, Shen-Zhen University, Shen-Zhen, China Correspondence: S-K. Huang (skhuang@nhri.org.tw)

Published online: 16 May 2018 